\title{
THE EXPRESSION OF CD38 ADP-RIBOSYL CYCLASE ECTOENZYME IN IMMUNE CELLS OF CARDIAC SURGICAL PATIENTS
}

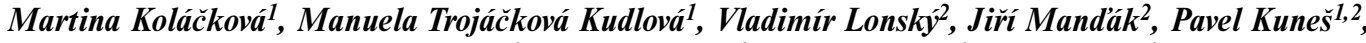 \\ Karolina Jankovičová ${ }^{1}$, Dana Vlášková ${ }^{2}$, Ctirad Andrýś, Jan Krejsek ${ }^{1}$
}

Charles University in Prague, Faculty of Medicine and University Hospital Hradec Králové, Czech Republic: Department of Clinical Immunology and Allergology ${ }^{1}$, Department of Cardiac Surgery ${ }^{2}$

\begin{abstract}
Summary: Background: This study was aimed at following the changes in the expression of CD38 ADP-ribosyl cyclase ectoenzyme on peripheral blood immune cells of patients undergoing cardiac surgical operations. Patients and Methods: The expression of CD38 on lymphoid and myeloid cells was determined by immunofluorescence and flow cytometry in forty cardiac surgical patients assigned to surgery either using ("on-pump", n=20) or without the use ("off-pump", n=20) of cardiopulmonary bypass. Results: There was a very rapid upregulation of CD38 expression in "on-pump” patients, becoming significant at declamping of aorta $(\mathrm{p}<0.01)$ for myeloid cells and at the weaning from $\mathrm{CPB}(\mathrm{p}<0.001)$ for lymphocytes. The increased expression of CD38 on lymphocytes in "off-pump" patients was prolonged for the entire observation period. However, significant differences in the expression of CD38 between "on-pump" and "off-pump" patients were not found either in lymphoid or myeloid cells. Conclusion: CD38 expression in immune cells of cardiac surgical patients is upregulated early during surgery, providing additional activation stimuli to the cell substrate of the inflammatory response induced by cardiac surgery.
\end{abstract}

Key words: Cardiac surgery; Immune cells; Activation; CD38; Expression

\section{Introduction}

Numerous events, which may results in the onset of an overt systemic inflammatory response, are inevitable induced in the course of cardiac surgery (12). Among these potentially harmful events, many stand out as the most clinically relevant to the very nature of surgical injury and the inevitable mechanical manipulation of the heart muscle. In addition, the contact of both humoral and cellular components of blood with artificial surfaces together with transient endotoxemia results in most cases from splanchnic hypoperfusion, and last but not least, from ischemia. Reperfusion injury of the heart and the lungs skew bodily regulations towards inflammation $(1,4,18)$. Massive, sometimes exaggerated, immune cell activation is a characteristic feature of this process. Cellular activation is inseparably linked to the calcium mobilisation in which the CD38 molecule is clearly involved. Indeed, it has now been firmly established that CD38 is a multi-functional enzyme catalyzing the metabolism of two distinct $\mathrm{Ca}^{2+}$ messengers, cyclic ADP-ribose (cADPR) and nicotinic acid adenine dinucleotide phosphate (NAADP) (14).

CD38 is a $42 \mathrm{kDa}$ type II transmembrane glycoprotein with its enzymatic active site located outside the cell (6).
Thus, CD38 is an extracellular enzyme or an ecto-enzyme, similar to some other leukocyte markers, including CD13, CD26, CD39, CD73 and CD157 (5). CD38, originally identified to be expressed in cells of lymphoid lineage and displaying stage - related variations, has much broader expression, regarding nowaday knowledge.

It is ubiquitously expressed in virtually all tissues. It is present not only on cell surfaces but also in various intracellular organelles, including the nucleus (3). It now appears that CD38 plays multiple roles in regulating the survival, activation, differentiation, migration and death of immunocytes (20). Indeed, CD38 expression profiles, as defined by staining with anti CD38 antibodies, are still widely used as diagnostic and prognostic markers for human diseases such as AIDS and chronic B-cells leukemias (17).

Our aims were to follow the dynamics of the expression of the CD38 molecule on lymphoid and myeloid cells in the peripheral blood of patients undergoing cardiac surgery either with ("on-pump") or without ("off-pump") the use of cardiopulmonary bypass. Another goal of our study was to discover if there are significant differences in the expression of CD38 between "on-pump" and "off-pump" patients. 


\section{Patients}

Forty patients ( 31 male, mean age $67.9 \pm 9$ years and 9 female, mean age $66.4 \pm 6.4$ years, collective mean age 67.6 \pm 8.5 years) referred for first-time coronary artery bypass grafting were enrolled in this study. Patients underwent either conventional myocardial revascularization with cardiopulmonary bypass and cardioplegic arrest of the heart ("on-pump", n=20, 16 male, 4 females, mean age $69.4 \pm 7$ years) or beating heart surgery ("off-pump", $n=20,15$ males, 5 females, mean age $65.9 \pm 9.7$ years). The patients were randomly assigned either to "on-pump" or to "off-pump" surgery by a member of the cardiac surgery staff outside the research team who was blinded to all variables pertinent to the study design.

Patients in both groups were comparable in age, preoperative left ventricular ejection fraction (median 0.65 in "on-pump", 0.65 in "off-pump" patients, respectively) and the number of performed coronary anastomoses (median 2.0 in “on-pump”, 2.0 in “off-pump”, respectively). All patients had been taking $100 \mathrm{mg}$ of aspirin in one daily dose, which was stopped five days before the operation. Patients treated with anti-inflammatory agents, either steroids or NSAID, were excluded from the study, as were patients with serum creatinine $\geq 130 \mu \mathrm{mol} / 1$ or with hepatic disorders. No patients were known to suffer from concomitant malignancies. Patients with active infectious diseases are not admitted to elective CABG in our department. The study protocol was approved by the Ethics Committee of the University Hospital in Hradec Králové. All active subjects had given informed written consent.

\section{Cardiac surgical procedure}

Cardiopulmonary bypass, "off-pump" technique and anesthesiological management have been recently described in detail elsewhere (13).

\section{Blood sampling}

Venous blood (peripheral venous blood from an antebrachial vein) was withdrawn in the operating room and during the postoperative period in the intensive care unit. Samples were collected into heparinized Vacutainer tubes manufactured by Becton Dickinson (NY, USA).

In "on-pump" patients, blood was withdrawn at following time points:

1: introduction to anaesthesia, which in both groups represented the baseline or reference value for all parameters measured thereafter:

1a: before cross-clamping of the aorta

1b: after aortic cross-clamp release

1c: after termination of CPB

2: after termination of the operation

3: the first postoperative day

4: the third postoperative day

5: the seventh postoperative day.
In “off-pump" patients, blood was withdrawn at:

1: introduction to anaesthesia

2: after termination of the operation

3: the first postoperative day

4: the third postoperative day

5: the seventh postoperative day.

\section{Methods}

Double immunofluorescence standard whole blood staining method was used. Briefly, $25 \mu 1$ of heparinized venous blood was incubated with the given pair of monoclonal antibodies $(2 \times 3 \mu 1)$ for 20 minutes at room temperature. After subsequent lysis (10 min) of red blood cell (Optilyse C, IMMUNOTECH, Marseille, France) samples, they were washed with buffered saline solution (PBS) and resuspended in PBS with azide. The following combinations of monoclonal antibodies were used: CD45-FITC/CD14-PE (LeukoGate) to distinguish between myeloid cells and lymphoid cells, isotypic control IgG1-FITC/IgG1-PE, CD45FITC/CD38-PE monoclonal antibodies which were purchased from IMMUNOTECH, Marseille, France. Measurements were performed using FACSCalibur flow cytometer and data acquired by CellQuest software (BD Bioscience, NY, USA). Lymphocytes and myeloid cells were identified on the basis of different CD45 v. CD14 expression (leukogate). No attempt was made to distinguish between granulocytes and monocytes. Results of flow cytometric analysis were expressed as a percentage of positive cells in the population of lymphocytes and in the population of myeloid cells.

\section{Statistical analysis}

We analyzed changes in the relative numbers of immune cells expressing CD38 in both groups of patients ("onpump", "off-pump"). Samples taken at the introduction to anesthesia were considered as a reference. Differences between "off- and on-pump" patients were also evaluated.

Data were analyzed using two-way ANOVA for repeated measures with Fisher LSD test and Dunnett test for multiple comparisons. To exclude the confounding effect of different ages and sexes in both groups, unpaired t-test and Fisher's exact test were performed.

A probability $(\mathrm{p})$ value $<0.05$ was considered significant.

Statistical analysis was performed with Statistica 5.5 software (Statsoft, USA).

\section{Results}

Expression of the CD38 molecule on lymphoid cells

In "on-pump" patients, the change in the expression of CD38 was already very rapid reaching significant increase $(p<0.001)$ at the weaning from CPB compared to preoperative levels. This increase continued through the finishing 
of surgery and the $1^{\text {st }}$ postoperative day, followed by a decline to the preoperative levels at the $3^{\text {rd }}$ and $7^{\text {th }}$ postoperative days (Fig. 1).

In "off-pump" patients, the expression of CD38 was significantly increased $(p<0.01)$ at the $1^{\text {st }}$ postoperative day and remained significantly increased for the rest of the observation period (Fig. 1).

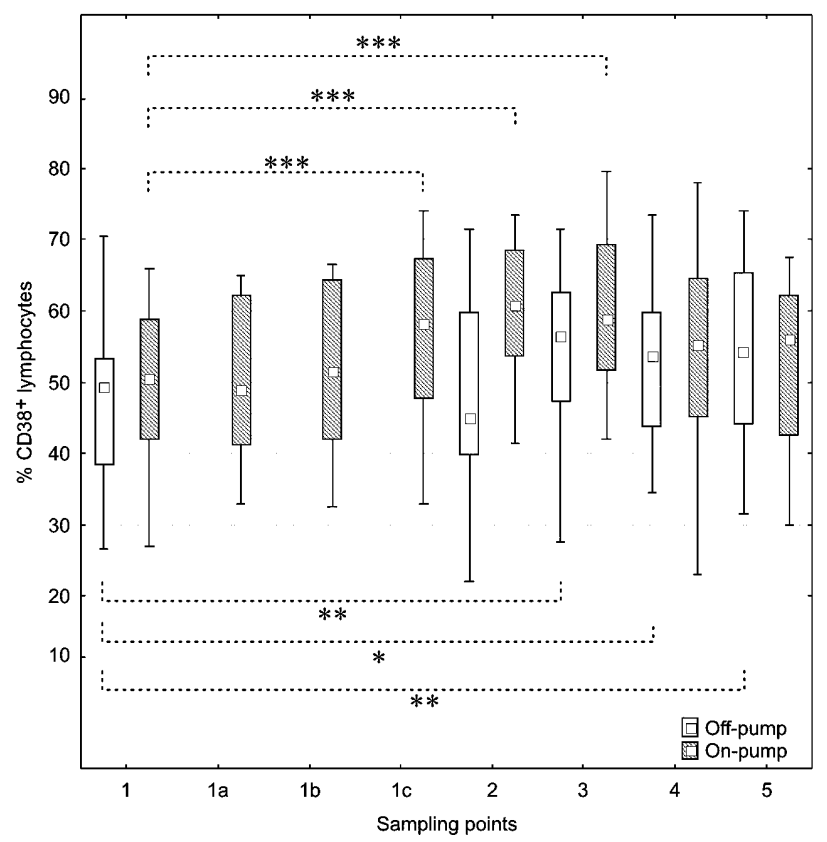

Fig. 1: Relative percentage of CD38 positive lymphocytes in cardiac surgical patients.

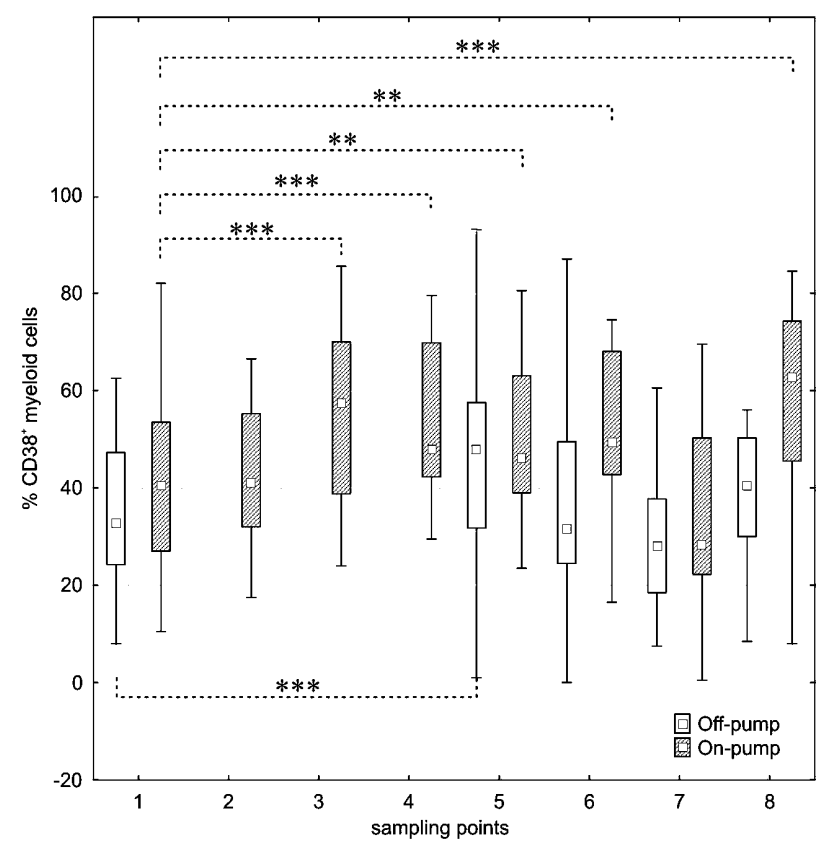

Fig. 2: Relative percentage of CD38 positive myeloids cells in cardiac surgical patients.
Comparing the dynamics of CD38 molecule expression between "on-pump" and "off-pump", a significant difference was not found. There was only a trend towards higher expression of the CD38 molecule in "on-pump" patients.

\section{Expression of the CD38 molecule on myeloid cells}

In "on-pump" patients the changes in the expression of CD38 in myeloid cells arrived even quicker, at declamping of the aorta, compared to lymphoid cells $(p<0.01)$. This increase persisted almost through the whole observation period except the $3^{\text {rd }}$ postoperative day (Fig. 2).

In "off-pump" patients, the dynamics of CD38 expression is rather monotonous compared to "on-pump" patients, being significantly higher only at the finishing of surgery $(\mathrm{p}<0.001)$. The percentage of CD38 expressing myeloid cells during the postoperative period was similar to the preoperative values (Fig. 2).

Comparing the dynamics of CD38 molecule expression between "on-pump" and "off-pump", a significant difference was not found. There was only a trend towards higher expression of CD38 molecule in myeloid cells in "onpump" patients.

\section{Discussion}

Broadly expressed multifunctional ectoenzyme CD38 is physically associated with cell surface receptors that mediate signalling cascades (14). It is seen early during differentiation of immune cells on various types of progenitor cells. Subsequently, its expression is heavily upregulated in immune cells after their appropriate stimulation. CD38 principally participates in metabolism of two distinct $\mathrm{Ca}^{2+}$ messengers, cyclic ADP ribose (cADPR) and nicotinic acid adenine dinucleotide phosphate (NAADP). The former is a cyclic nucleotide that modulates the ryanodine receptor and mobilizes the endoplasmic $\mathrm{Ca}^{2+}$ stores at neutral or alkaline $\mathrm{pH}$. NAADP is structurally distinct from cADPR and targets separate $\mathrm{Ca}^{2+}$ stores, for example, acidic organelles like lysosomes (14).

CD38 regulates inflammatory responses by modulating the activity of both leukocytes and nonhaemopoietic cells in the inflammed tissue. Enzymatic generation of cADPR by $\mathrm{CD} 38$ regulates intracellular calcium release and calcium influx during neutrophil activation, including their adhesion and chemotaxis (2). This is very relevant to our cardiac patients, as their blood cells granulocytes are heavily stimulated during surgery both by the contact with artificial surfaces in "on-pump" patients or by numerous proinflammatory stimuli that are raised in all cardiac surgical patients irrespective of the surgical strategy used.

Many parameters of granulocytes describing their activation status have already been identified and published. Upregulation in the expression of an activated form of leukocyte $\beta_{2}$ chain integrin CD18 recognized by the MEM-148 monoclonal antibody was found in our previous study (11). Another activation marker of phagocytic cells, high affinity 
receptor for $\mathrm{Fc}$ part of $\mathrm{IgG}$ immunoglobulins (Fc $\gamma \mathrm{RI}$, CD64) determined by a novel quantitative approach utilizing Leuko64 ${ }^{\mathrm{TM}} \mathrm{kit}$, is also induced by cardiac surgery, as we have shown (9).

We believe, in accordance with the suggestion of Partida-Sanchez et al (20) who utilized infection in the modelling of CD38 activity, that inflammatory cells such as neutrophils constitutively express low levels of CD38 on their surfaces. However, under normal conditions in vivo, neutrophils produce only very small quantities of cADPR because the substrate $\left(\mathrm{NAD}^{+}\right)$is limiting (7). If neutrophils encounter free $\mathrm{NAD}^{+}$in their extracellular environment, as might be expected if cell damage has occurred, then the neutrophils will be able to produce cADPR in much larger quantities. Increased cADPR will then allow the neutrophils to respond to various danger signals, including chemoattractive compounds, by activating plasma membrane ion channels. This sustained calcium signal will allow the neutrophils to activate and migrate. When injury or infection is resolved, extracellular NAD ${ }^{+}$levels would return to baseline, cADPR production by the neutrophil would be reduced and proinflammatory signalling throught the various surface receptors would be attenuated.

The physiological functions of CD38 are clearly widespread. Not only innate immunity in its inductory and regulatory arms but also adaptive immune response is regulated by the CD38 molecule $(15,19)$. Regarding its role in the differentiation and activation in B cells lineage antigen, the driven immune response of mature B cells is influenced by the activity of CD38. Such cells express CD38 and undergo a few rounds of replication before being recruited to germinal centres within secondary follicles. These cells then undergo the process of isotypic switching and somatic hypermutation in this microenvironment. CD38 expression is now appreciated as a prognostic marker in some B lymphoproliferation disorders (16).

CD38 functions in T cells are mediated by cell surface association with TcR CD3 complex receptors and other membrane molecules. Little expression of CD38 is seen in the resting T cells. However, CD38 is rapidly upregulated upon lymphocytes activation, as also evident from our results. CD38 ligation induces activation of transcription factors involved in the development of inflammatory responses, such as NFKB. Activation of cells via CD38 leads to increased production of cytokines, especially INF $\gamma$ by $\mathrm{T}$ cells (22). Cardiac surgery also leads to $\mathrm{T}$ cell activation (8). In our previous work, the percentage of HLA DR expressing lymphocytes in "on-pump" patients was significantly higher compared to "off-pump" patients on the $1^{\text {st }}$ and $2^{\text {nd }}$ postoperative days (8). It was a surprise for us to see that the number of CD38 positive lymphocytes in "offpump" patients was significantly increased through the entire observation period in comparison to "on-pump" patients in whom the number of CD38 positive cells was already returning back to the preoperative value on the $3^{\text {rd }}$ postoperative day.
In conclusion, we are in harmony with the opinion of others utilizing infection as a model of inflammation (20). We propose that CD38 in cardiac surgical patients works in a fashion ressembling surveillance receptors such as Toll-like receptor activity (10). When cell damage occurs, ecto-enzyme CD38 is activated by increased substrate availability and produces significant quantities of calcium - mobilizing metabolites that can modulate proinflammatory activities of a complex cell substrate of inflammation raised in cardiac surgical patients by the presence of danger signals (23). When the cellular damage has been controlled, metabolites produced by CD38 molecule activity regulating calcium mobilization in cells are reduced and proinflammatory activities of cells are attenuated. CD38 does not recognize danger signals directly as regular pattern recognition receptors do. Instead, CD38 acts essentially as a sensor for endogenous cell damage and necrosis.

CD38 is now the focus of interest as a promising target to modulate inflammatory response (21). Several compounds with the potential to modulate CD38 activity are now being tested exploiting in vitro and animal models. Based on the substantial progress in this field, it is plausible that CD38 agonists could enrich therapeutical armamentarium of all physicians coping with undesired inflammatory response in their patients, including cardiac surgeons.

\section{Acknowledgment}

This work was supported by Ministry of Education, Czech Republic, project No. MSM0021620812 and Internal Grant Agency of Ministry of Health, Czech Republic, No. NR/9090-4.

Authors are in debt to Jaroslav Kubíček and Martin Volt, perfusionists both in the staff of Dept. of Cardiac Surgery for their valuable support to this study.

\section{References}

1. Ascione R, Lloyd CT, Underwood MJ, Lotto AA, Pitsis AA, Angelini GD: Inflammatory response after coronary revascularization with or without cardiopulmonary bypass. Ann thorac Surg 2000;69:1198-2004

2. Czura AW, Czura ChJ. CD38 and CD157: Biological observations to clinical therapeutic targets. Mol Med 2006;12(11-12):309-11.

3. Fernandez JE, Deaglio S, Donati D et al. Analysis of the distribution of human CD38 and of its ligand CD31 in normal tissues. J Biol Regul Homeostatic Agents 1998; 12:81-91.

4. Franke A, Lante W, Fackeldey V et al. Pro-inflammatory cytokines after different kinds of cardio-thoracic surgical procedures: is what we see what we know? Eur J Cardio-thoracic Surgery 2005;28:569-75.

5. Goding JW, Howard MC. Ecto-enzymes of lymphoid cells. Immunol Rev 1998; 161:5-10.

6. Howard M, Grimaldi JC, Bazan JF et al. Formation and hydrolysis of cyclic ADPribose catalyzed by lymphocyte antigen CD38. Science 1993;262:1056-9.

7. Kim UH, Kim JS, Han MK, Park BH, Kim HR. Purification and characterization of NAD glycohydrolase from rabbit erythrocytes. Arch Biochem Biophys 1993; 305:147-52.

8. Koláčková M, Jankovičová $\mathrm{K}$, Trojáčková Kudlová $\mathrm{M}$ et al. The effect of cardiac surgery on peripheral blood lymphocyte populations. Acta Med (submitted).

9. Koláčková M, Kudlová M, Kuneš P et al. Early expression of FçRI (CD64) on monocytes of cardiac surgical patients and higher density of monocyte antiinflammatory scavenger CD163 receptor in "on-pump” patients. Med Inflam 2007 (in press).

10. Krejsek J, Kuneš P, Koláčková $M$ et al. The number of Toll-like receptor 2 and 4 expressing immune cells is changed during cardiac surgical operation. Scand $\mathbf{J}$ Clin Lab Invest (submitted) 
11. Kudlová M, Koláčková $\mathrm{M}$, Kuneš $\mathrm{P}$ et al. Expression of an activated form of integrin $\beta_{2}$ chain CD18 in cardiac surgical operations. Acta Med 2007;50(3): 187-193.

12. Kunes P, Lonsky V, Mandak J et al. The inflammatory response in cardiac surgery. An up-to-date overview with the emphasis on the role of heat shock proteins (HSPs) 60 and 70. Acta Med 2007;50(2):93-99.

13. Kunes P, Lonsky V, Mandak J, Kolackova, M., Andrys, C., Kudlova, M., Krejsek, J. The long pentraxin 3 in cardiac surgery: distinct responses in "on-pump" and "off-pump" patients. Scand Cardiovasc J 2007;41(3):171-9.

14. Lee HCh. Structure and enzymatic functions of human CD38. Mol Med 2006;12(11-12):317-23

15. Malavasi F, Deaglio S, Ferrero E et al. CD38 and CD157 as receptors of the immune systém: a bridge between innate and adaptive immunity. Mol Med 2006 12(11-12):334-41.

16. Montserrat E. New prognostic markers in CLL. Hematology Am Soc Hematol Educ Program 2006;279-84.

17. Morabito F, Damle RN, Deaglio S, Keating M, Ferrarini M, Chiorazzi N.
The CD38 ectoenzyme family: advances in basic science and clinical practice. Mol Med 2006;12(12-12):342-4.

18. Paparella D, Yau TM, Young E. Cardiopulmonary bypass induced inflammation: pathophysiology and treatment. An update. Eur J Cardio-thoracic Surgery 2002; 21:232-44.

19. Pfister M, Ogilvie A, da Silva ChP, Grahnert A, Guse AH, Hauschildt S. NAD degradation and regulation of $\mathrm{CD} 38$ expression by human monocytes/macrophages. Eur J Biochem 2001;268:5601-8.

20. Sanchez SP, Randall TD, Lund FE. Innate immunity is regulated by CD38, an ecto-enzyme with ADP-ribosyl cyclase activity. Microbes Infect 2003;5:49-58.

21. Stevenson GT. CD38 as a therapeutic target. Mol Med 2006;12(11-12):345-6.

22. Viegas MS, do Carmo A, Silva T et al. CD38 plays a role in effective containment of mycobacteria within granulomata and polarization of Th1 immune responses against Mycobacterium avium. Microbes Infect 2007;9:847-54.

23. Wehlin L, Vedin J, Vaage J, Lundahl J. Peripheral blood monocyte activation during coronary artery bypass grafting with or without cardiopulmonary bypass. Scand Cardiovasc J 2005;39:78-86.

Submitted February 2008.

Accepted March 2008.

\section{Corresponding author:}

Prof. RNDr. Jan Krejsek, CSc., Department of Clinical Immunology and Allergology, University Hospital, Sokolská 581, 50005 Hradec Kralové, Czech Republic, e-mail: krejsek@fnhk.cz 